El Segundo sexo de Simone de Beauvoir analizado por Susana López Pavón

The second sex of Simone de Beauvoir analyzed by Susana López Pavón

\title{
O segundo sexo de Simone de Beauvoir analisado por Susana López Pavón
}

\section{Luis Cibanal Juan}

Profesor Profesor Honorífico de la Facultad de Ciencias de la Salud de la Universidad de Alicante.

Cómo citar esta reseña en edición digital: Cibanal Juan, L. (2020). Reseña de Simone de Beauvoir. El Segundo sexo: Lectura crítica de la introducción y la conclusión. de Susana López Pavón. Cultura de los Cuidados (Edición digital), 24(56). Recuperado de http://dx.doi.org/10.14198/cuid.2020.56.23

Correo electrónico: luis.cibanal@ua.es Correspondencia: Remitirse al correo electrónico. Recibido/ Aceptado: Reseña Invitada.
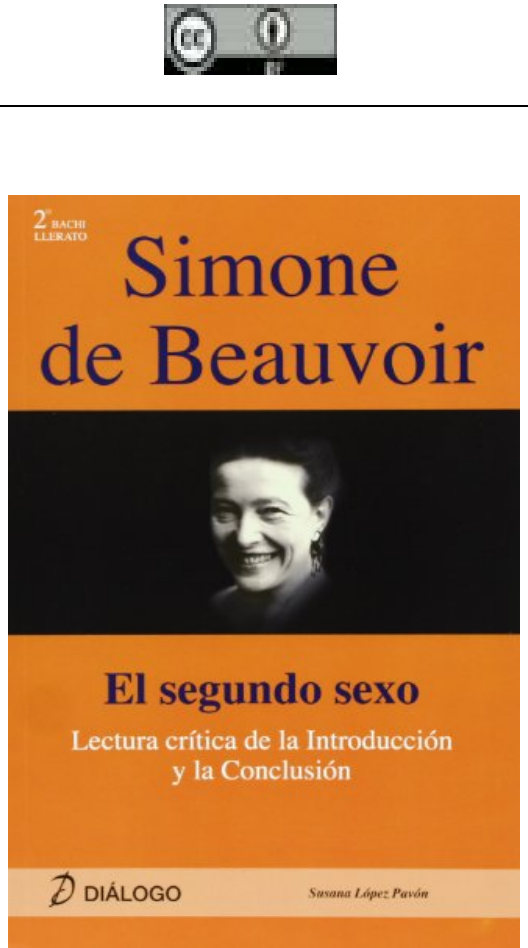

López, Pavón, S. (2012) Simone de Beauvoir. El Segundo sexo: Lectura crítica de la introducción y la conclusión. Madrid: Ed. Diálogo. ISBN 10: 8496976688 ISBN 13: 9788496976689 


\section{ABSTRACT}

Review of the book "Simone de Beauvoir. The Second Sex: A Critical Reading of the Introduction and Conclusion" by Susana López Pavón.

\section{RESUMEN}

Reseña de la obra "Simone de Beauvoir. El Segundo sexo: Lectura crítica de la introducción y la conclusión" de Susana López Pavón.

\section{RESUMO}

Revisão da obra "Simone de Beauvoir. The Second Sex: A Critical Reading of the Introduction and Conclusion" de Susana López Pavón.

El libro que nos presenta la autora sobre Simone de Beauvoir nos da, en primer lugar, un breve resumen sobre la biografía de Simone de Beauvoir, así como el contexto filosófico en el que se desenvuelve, que es el existencialista. En segundolugar, nos da unas breves pinceladas sobre las ideas feministas de la Ilustración al siglo XXI.

La obra: El Segundo Sexo aparece de manera completa en 1949, y aunque en esta obra se plantean los temas básicos del movimiento feminista que cobra fuerza sobre todo en los años 70 , su autora no se identifica en esta época como feminista. El ensayo surge como necesidad personal de reflexión sobre lo que para ella misma había significado ser mujer, pero alcanza proporciones mucho más amplias, acerca de la condición femenina en general.

El Segundo Sexo está considerado como la obra feminista más importante del siglo XX, referencia ineludible tanto para profundizar en sus planteamientos como para polemizar con ellos. Considera como interlocutores de El Segundo Sexo el psicoanálisis (Freud) y el materialismo histórico (Marx), haciendo un repaso somero sobre algunos de los aspectos principales de estas dos teorías filosóficas que Beauvoir tiene muy en cuenta en su investigación, rechazando el determinismo del comportamiento humano que las dos teorías afirman.

Simone de Beauvoir se identifica con el existencialismo ateo francés cuyo punto de partida puede situarse en la publicación en 1943 de El Ser y la nada de Jean Paul Sartre. En El Segundo Sexo afirma que la perspectiva que adopta es la de Heidegger, Satre y Merleau-Ponty. Así mismo, hace un breve resumen de la filosofía existencialista. Simone de Beuavoir, Realiza una Hermenéutica propia del existencialismo, entendiendo éste como el conjunto de elementos propios que la filosofía desarrolla en la interpretación (hermenéutica) que realiza de la corriente filosófica existencialista.

Realiza preguntas que tienen la virtualidad de hacer temblar los cimientos de significado que parecían sólidamente anclados. Este el caso de la pregunta que inicia la reflexión en El segundo sexo: “QQué es una mujer?”. Lo que hace nuestra autora al formular la pregunta es llevar una duda radical al significado del concepto "mujer". Esta pregunta planteada de manera explícita sitúa la duda sobre un concepto cuyo significado parecía ya establecido de manera firme, encorsetando a los individuos concretos a los que se aplica. Este libro, aunque es muy breve, pues es un resumen de los grandes temas que aborda Simone de Beauvoir en El Segundo sexo, sin embargo es muy ameno y nos hace reflexionar por ejemplo sobre: ¿cómo se ha constituido, cómo ha evolucionado a lo largo del tiempo y qué discursos han contribuido a la 
consolidación de la feminidad? El punto de partida es la constatación de la falta de simetría entre feminidad y masculinidad en la mayor parte de las sociedades. Lo masculino se ha identificado con lo humano y lo femenino se ha constituido como "carencia" de determinadas cualidades respecto a lo masculino. Estas supuestas carencias se asocian a la sexualidad específica de las mujeres, como si el cuerpo de la mujer estuviese "lastrado" por ella y fuese defectuoso en comparación con el varón. "La humanidad es masculina y el hombre define a la mujer no por ella misma, sino en relación con él”. En relación a esta falta de simetría, lo que Beauvoir se plantea averiguar es qué circunstancias la hicieron posible, es decir, por qué la mujer se determina y se diferencia respecto al hombre y no a la inversa, por qué el varón es tradicionalmente considerado Sujeto y ella no: "Ella se determina y se diferencia con relación al hombre. Lo opuesto no es válido: ella es lo inesencial frente a lo esencial. Él es el Sujeto, el Absoluto: ella es la alteridad". ¿

¿Por qué las relaciones entre hombres y mujeres no se plantearon en términos de reciprocidad? Si toda conciencia tiende a reconocerse como tal afirmándose como sujeto frente a las demás conciencias, ¿cómo es posible que haya triunfado un punto de vista que anula la consideración de sujeto para, aproximadamente la mitad de la humanidad? “ ¿De dónde le viene a la mujer esta sumisión”? Esta es la pregunta que clarifica la mirada que se desarrolla en la primera parte de la investigación, que coincide con el primer volumen de $E l$ segundo sexo, titulado: "Los hechos y los mitos". Es una mirada regresiva porque trata de entender la opresión de las mujeres clarificando las circunstancias que confluyeron en el pasado para que esta situación injusta se gestase. Es una mirada analítica porque descompone el fenómeno universalmente extendido de la opresión de las mujeres en diferentes aspectos, para que se muestre en su complejidad y pueda apreciarse cómo su consolidación y evolución se debe a la confluencia de elementos muy distintos que la mantienen y la justifican.

Aborda otros puntos como: Realizar una lectura feminista de la dialéctica hegeliana del amor y el esclavo. La mujer como la "Otra” en la sociedad patriarcal. La falta de simetría entre hombres y mujeres. Lectura feminista del amo y el esclavo. Las dificultades para abolir la relación de vasallaje. La ambigüedad de la situación de las mujeres. El factor cultural como factor decisivo en el análisis de las causas de la opresión de la mujer. El cuerpo humano como cuerpo vivido. Rechazo del determinismo biológico. La evolución de la condición de las mujeres. La socialización desigual. La "mujer, mujer" como producto artificial. La educación y evolución colectivas para conseguir la autonomía de las mujeres $\mathrm{y}$ la reciprocidad de las relaciones entre hombres y mujeres, etc.

En definitiva, hemos visto a través de estas pinceladas cómo es un libro que nos hace pensar y cuestionarnos, por lo que creo que merece la pena dedicarle unas horas a su lectura.

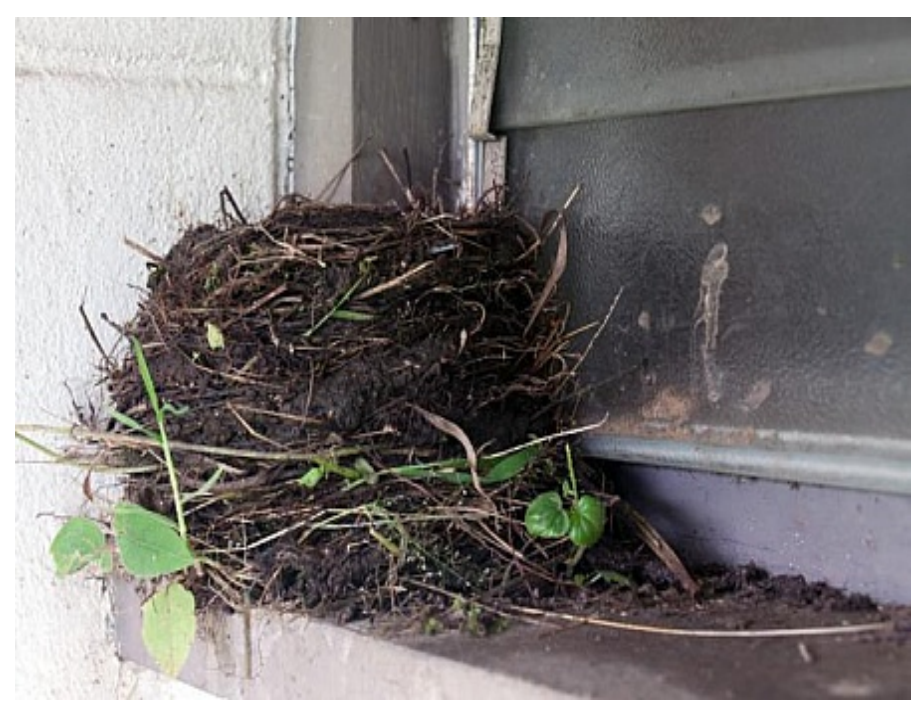

Fuente: https://freerangestock.com/photos/39634/nest.html 